\title{
Broad-band polarization conversion from a finite periodic structure in the microwave regime
}

\author{
B. T. Hallam and C. R. Lawrence \\ QinetiQ Ltd, Cody Technology Park, Farnborough, Hampshire GU14 OLX, United Kingdom \\ I. R. Hooper and J. R. Sambles \\ Thin Film Photonics, School of Physics, University of Exeter, Exeter EX4 4QL, United Kingdom
}

(Received 29 September 2003; accepted 8 December 2003)

\begin{abstract}
A reflection grating demonstrating extraordinarily broad-band polarization conversion in a nondiffractive regime has been studied at microwave frequencies. This single-element structure has been fabricated by electrolessly plating a metallic layer onto a stereo-lithographically produced resin profile. Angle-dependent microwave reflectivity data collected from the grating indicates polarization conversion of greater than $80 \%$ over a spectral bandwidth equivalent to the entire visible regime (factor of 2 in frequency). This supports an earlier publication in which it was predicted that a broad-band polarization converter could be created from a suitably profiled diffraction grating. (C) 2004 American Institute of Physics. [DOI: 10.1063/1.1645661]
\end{abstract}

A metallic grating with a periodicity of less than onehalf of the wavelength of incident radiation, $\lambda_{0}$, has no real diffracted orders in the incident half-space. Such a grating is thereby termed zero-order or nondiffractive. The surface morphology of such a structure does, however, give rise to form birefringence, the uniaxial properties of which are well documented in the visible and microwave regimes. ${ }^{1-3}$

The degree of electromagnetic anisotropy exhibited by any subwavelength periodic surface is determined by the detailed nature of the surface topology. Such structures are normally severely limited in their operational bandwidth, and hence are largely restricted to application-specific activities which require optimal performance for a specified frequency and/or orientation of the sample.

With a metal surface, a second mechanism for inducing polarization conversion is via excitation of resonant phonon or plasmon modes. ${ }^{4-6}$ In the latter case, a corrugated surface is commonly employed to enable coupling to the surface plasmon-polariton (SPP) modes in the nonclassical mount ${ }^{5,6}$ (i.e., grating grooves not perpendicular to the plane of incidence). Here again, the excitation of such a mode is regarded as being highly dependent on the precise nature of the grating boundary and is generally also narrow banded.

Recently, however, it has been shown that in the visible regime a suitably designed grating will produce polarization conversion over a very broad frequency range. ${ }^{7}$ The crux of the idea presented in Ref. 7 is that through an appropriate choice of grating parameters, the two mechanisms highlighted above, form birefringence and the excitation of surface resonant modes, may combine to give polarization conversion over a broad spectrum of incident frequencies.

Manufacture of such a structure for visible wavelengths is relatively elaborate due to the submicron accuracy that is required. However, it is easier to demonstrate the effect in the microwave regime. Since the source of the form birefringence is primarily geometrical in character, then changing wavelength is primarily, for this effect, a scaling exercise. Equally, coupling to the surface plasmon and the dispersion curve for deep (depth greater than 0.1 pitch) gratings will also be dominated by geometry. Thus the essential ideas for the visible domain are still valid at microwave frequencies, with the additional benefit that metals at these frequencies do not experience inter- or intraband absorption loss mechanisms.

For the purpose of this study, a grating of area $900 \mathrm{~cm}^{2}$ was fabricated in resin via standard stereo-lithographic techniques. $^{8,9}$ A 3D System's SLA5000 imaging instrument with solid state laser and SmartSweep ${ }^{\mathrm{TM}}$ technology was used to convert the 3D CAD model of the desired structure into a solid resin duplicate via photopolymerization. The precise geometry of the chosen structure was determined by scaling the designs of Ref. 7 to a center frequency of 14 GHz. The design chosen was a periodic array of Gaussianpeaked ridges, described with a pitch $\left(\lambda_{g}\right)$ equal to 14.85 $\mathrm{mm}$, a ridge height of $9.3 \mathrm{~mm}$ and ridge width full width at half maximum (FWHM) of $0.19 \mathrm{~mm}$.

Metallization of the grating was performed using a QinetiQ proprietary process based on electroless deposition. ${ }^{10,11}$ This was used to deposit a copper film with a thickness of $\sim 2 \mu \mathrm{m}$ and near-bulk conductivity. ${ }^{10,11}$

The grating was positioned on the center of rotation of a rotating table as shown in Fig. 1. Parabolic reflectors were used to provide a parallel beam at the sample and also to

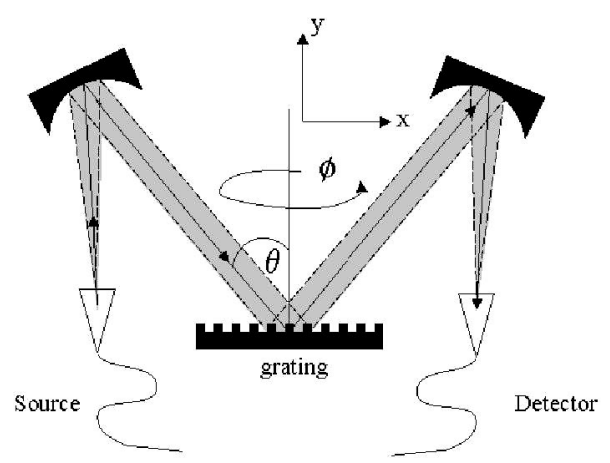

FIG. 1. Experimental apparatus used for collection of angle-dependent reflectivity data. 

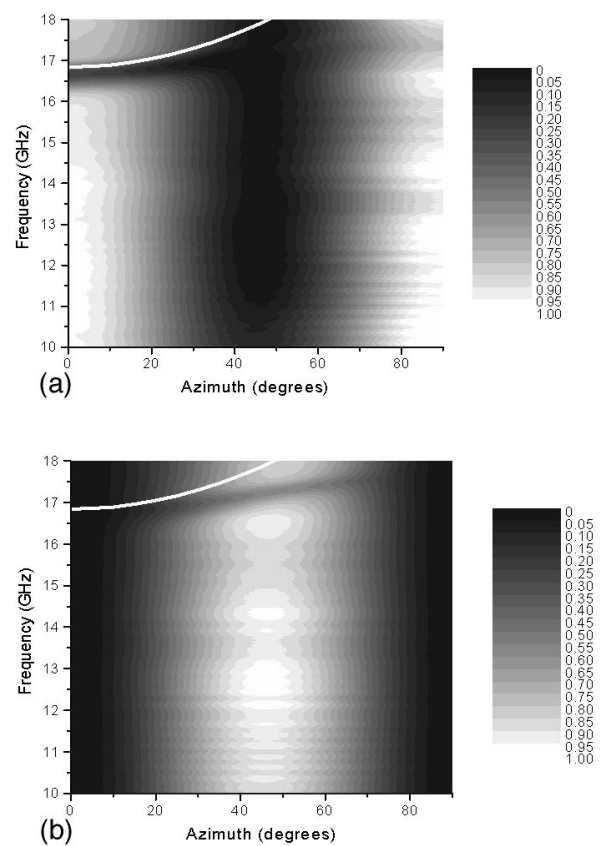

FIG. 2. (a) Experimental reflectivity data from the grating, with polarization conserved $\left(R_{\mathrm{pp}}\right)$. The solid white line marks the diffraction edge, above which power is redirected into a diffracted order. (b) Experimental reflectivity data from the grating, with polarization converted $\left(R_{\mathrm{ps}}\right)$.

focus the beam reflected from the sample into the detector. An HP 8757D scalar network analyzer was used to record angle-dependent reflectivity data from the sample as a function of azimuthal angle for frequencies in the range 10-18 GHz. The polarization of the incident and detected signals was selected by suitable orientation of the source and detector horns. The $p$-polarized radiation (Transverse Magnetic, $\mathrm{TM})$ has the electric vector in the plane of incidence, and $s$-polarized (Transverse Electric, TE) radiation has the electric vector perpendicular to the plane of incidence. Repeating the experiment with a planar metal sheet enabled the normalization of the results.

Additional measurements in a standard NRL $\operatorname{arch}^{12}$ were then conducted on the grating at a set azimuthal angle of $45^{\circ}$ in order to determine how many periods of the grating were required in order to produce significant polarization conversion. Finite gratings were produced by covering the outlying grooves with a thin $(0.2 \mathrm{~mm})$ flat aluminum overlay to simulate a planar border to the centrally placed grating region. The finite grating was placed at the center of the anechoic arch and microwave reflectivity data were obtained from bistatic measurements with Flann Microwave DP240 lensed antennas.

The graphs in Figs. 2(a) and 2(b) present typical experimental angle-dependent reflectivity traces for the polarization conserved $\left(R_{\mathrm{pp}}\right)$ and polarization converted $\left(R_{\mathrm{ps}}\right)$ signals recorded at an incident angle of $11.5^{\circ}$. Here the first subscript denotes the incident polarization and the second subscript denotes the detected polarization. The curved line in the top-left corner of each graph marks the boundary of the nondiffractive regime: above this line, the frequencies are high enough to give rise to diffractive effects, and hence this line is defined to be the critical edge. Minor anomalies can be seen in the polarization-conserved data at low frequencies and azimuthal angles near $90^{\circ}$ : these are normalization diffiDownloaded 27 May 2008 to 144.173 .6 .22 . Redistribution subject to
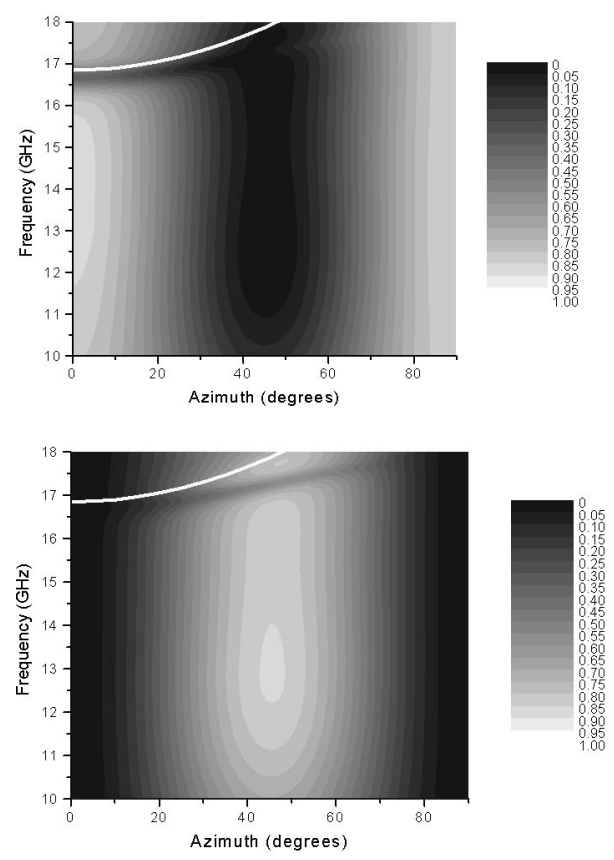

FIG. 3. Modeled reflectivity of the grating, with polarization conserved $\left(R_{\mathrm{pp}}\right)$; modelled reflectivity of the grating, with polarization converted $\left(R_{\mathrm{ps}}\right)$.

culties, and are due to experimental limitations regarding beam divergence.

The graphs exhibit a broad minimum in the polarizationconserved signal and a corresponding maximum in the polarization converted signal. As expected, the polarization conversion is at a maximum at a grating azimuth of $45^{\circ}$, but demonstrates over $80 \%$ conversion of the signal for an angular range of greater than $15^{\circ}$, and covers the entire recorded spectrum $(10-18 \mathrm{GHz})$. This continuous band is broken at $\sim 17 \mathrm{GHz}$, with a second feature observed running across the data (starting at $\sim 16.75 \mathrm{GHz}$ at zero azimuth, lying approximately parallel to the critical edge). This absorption is associated with the coupling of radiation into an SPP mode. The appearance of the SPP at this energy arises because the Gaussian surface profile contains a large, out-ofphase, first harmonic component. This leads to a band-gap opening in the SPP dispersion curve at normal incidence, to which the low-energy branch may be coupled. ${ }^{7}$

These results agree well with modeled predictions from a detailed optical theory ${ }^{13}$ based on the differential formalism of Chandezon et al., ${ }^{14}$ as shown in Fig. 3. During the modeling process, the measured parameters describing the grating profile and experimental orientation were kept fixed, while the permittivities of the metal were varied in order to obtain the closest comparison to the experimental data. The modeled parameters were:

Gaussian ridge heights: $9.3 \mathrm{~mm}$,

Gaussian ridge widths: 0.19 FWHM,

beam angle spread: $2 \mathrm{deg}$,

metal film thickness: $1 \mu \mathrm{m}$,

incident angle: $11.5 \mathrm{deg}$,

pitch: $14.85 \mathrm{~mm}$,

with the metal permittivities obtained for this closest fit being $\varepsilon_{r}=-1 \times 10^{-3}$ and $\varepsilon_{i}=3 \times 10^{5}$.

For comparison purposes, numerical modeling was also performed on a pure sinusoid grating of equal pitch (Fig. 4)
AIP license or copyright; see http://apl.aip.org/apl/copyright.jsp 


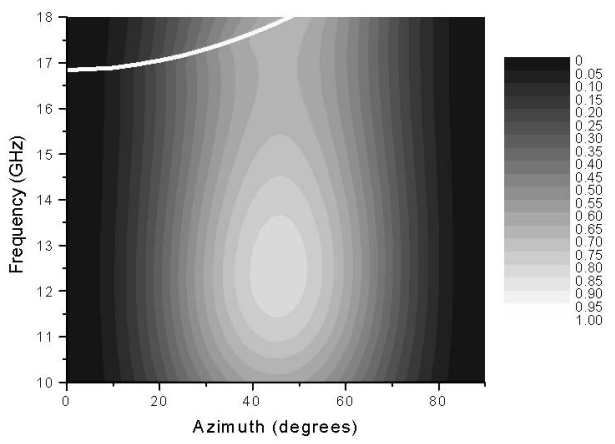

FIG. 4. The predicted polarization conversion from a sinusoidal grating of the same groove depth and pitch $\left(R_{\mathrm{ps}}\right)$.

illuminated at the same angle of incidence. The results in Fig. 4 show polarization conversion occurring only over a greatly reduced spectral range for this sinusoidal profile in comparison with the Gaussian-peaked sample. It is thus abundantly clear that the groove shape is crucial in the design of an optimized polarization converter.

Figure 5 shows the effect of limiting the number of grating grooves on the performance of the polarization conversion signal. The data show that as few as three grooves produce a polarization conversion level of $\sim 50 \%$, and that only five grooves are required to achieve $>95 \%$ polarization conversion at the central wavelength of operation. This suggests that reasonable performance may be obtained from such a

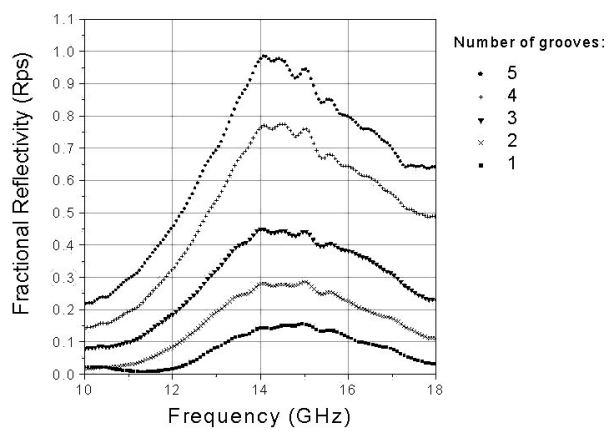

FIG. 5. Polarization conversion signal $\left(R_{\mathrm{ps}}\right)$ as a function of frequency for different numbers of grooves (see text for details). device even in applications that prohibit the creation of a seemingly infinite (large area) grating profile. The spectral breadth of the polarization conversion is seen to increase as the number of periods in the structure is reduced. This is most probably due to the variation of beam spot size with frequency. A similar result was obtained in Ref. 3.

A single element broadband polarization conversion structure has been demonstrated in the microwave regime. Angle-dependent reflectivity data have been recorded which demonstrates more than $80 \%$ polarization conversion over a frequency range greater than $10-18 \mathrm{GHz}$ (and $>90 \%$ over the majority of this region). These results agree well with numerical modeling. In addition, it is found that as few as four grating periods are required to produce $>70 \%$ polarization conversion over a narrow frequency band, suggesting that such a structure could be utilized in applications where large-area samples are prohibited.

This work has verified the predicted performance of the optimized surface profile, and hence supports the premise that such structures could be used to produce broadband polarization converters.

This work was conducted under the Materials Technology Domain of the UK Ministry of Defense Corporate Research Program.

${ }^{1}$ M. Born and E. Wolf, Principles of Optics (Pergamon, New York, 1980).

${ }^{2}$ S. Cornbleet, Microwave Optics (Academic, New York, 1976).

${ }^{3}$ C. R. Lawrence and A. S. Treen, IEE Proc. Microw. Antennas Propag. 146, 241 (1999).

${ }^{4}$ R. W. Morse, H. V. Bohrn, and J. D. Gavenda, Phys. Rev. 109, 1394 (1958).

${ }^{5}$ G. P. Bryan-Brown, J. R. Sambles, and M. C. Hutley, J. Mod. Opt. 37, 1227 (1990).

${ }^{6}$ S. J. Elston, G. P. Bryan-Brown, T. W. Priest, and J. R. Sambles, Phys. Rev. B 44, 3483 (1991).

${ }^{7}$ I. R. Hooper and J. R. Sambles, Opt. Lett. 27, 2152 (2002).

${ }^{8}$ J. Jakubiak and J. F. Rabek, Polimery 45, 759 (2000).

${ }^{9}$ J. Jakubiak and J. F. Rabek, Polimery 46, 164 (2001).

${ }^{10}$ W. Damerell, S. Appleton, D. Johnson, and G. Fixter, UK Patent No. GB, 01, 134, 08 (2001).

${ }^{11}$ W. Damerell, S. Appleton, D. Johnson, and G. Fixter, UK Patent No. GB, 01, 285, 71 (2001).

${ }^{12}$ E. F. Knott, J. F. Shaeffer, and M. T. Tuley, Radar Cross Section (Artech House, Boston, 1993).

${ }^{13}$ N. P. K. Cotter, T. W. Priest, and J. R. Sambles, J. Opt. Soc. Am. 12, 1097 (1995).

${ }^{14}$ J. Chandezon, M. T. Dupuis, G. Cornet, and D. Maystre, J. Opt. Soc. Am. 72, 839 (1982). 